\title{
A case of arrhythmogenic right ventricular cardiomyopathy with biventricular involvement
}

\author{
Filippo Brandimarteㄹ, Alessandro Battagliese' ${ }^{1}$, Silvana Petronilla Pirillo², Maria Teresa Mallus ${ }^{3}$, \\ Rosa Maria Manfredi ${ }^{3}$, Giovanni Carreras ${ }^{1}$ \\ ${ }^{1}$ Department of Medicine, Cardiology and Rehabilitation Unit, San Giovanni Addolorata Community Hospital, Rome; \\ ${ }^{2}$ Department of Radiology, San Giovanni Addolorata Community Hospital, Rome; ${ }^{3}$ Department of Emergency, CCU \\ and Interventional Cardiology, San Giovanni Addolorata Community Hospital, Rome, Italy
}

\begin{abstract}
We reported a case of a young adult male aged 18 years admitted in our institution for syncope during a basketball match. No previous symptoms were reported. Electrocardiogram (ECG) showed T-wave inversion in the anterior leads and an incomplete right bundle branch block. Surprisingly, a complete echocardiographic evaluation demonstrated the presence of severe right ventricular enlargement with significant wall motion abnormalities, apical aneurysm and reduced systolic function. Cardiac Magnetic Resonance was pathognomonic for a fibro-fatty replacement of both ventricles. We decided for a subcutaneous defibrillator implantation and, after inducing a ventricular fibrillation to test the device status, epsilon wave appeared on the ECG. This clinical scenario depicted an advanced arrhythmogenic right ventricular cardiomyopathy at its first clinical manifestation.
\end{abstract}

\section{Case Report}

An 18-year-old male from the Philippines arrived at the ED

\begin{abstract}
Correspondence: Filippo Brandimarte, Department of Medicine, Cardiology and Rehabilitation Unit, San Giovanni Addolorata Community Hospital, Via dell'Amba Aradam 9, 00184 Roma, Italy. E-mail: brandimarte.filippo@gmail.com
\end{abstract}

Key words: Syncope; arrhythmogenic right ventricular cardiomyopathy; sudden death.

Contributions: All authors participated equally to the preparation of the manuscript and had full access to the data.

Received for publication: 16 November 2018.

Accepted for publication: 25 February 2019.

CCopyright F. Brandimarte et al., 2019

Licensee PAGEPress, Italy

Monaldi Archives for Chest Disease 2019; 89:1009

doi: 10.4081/monaldi.2019.1009

This article is distributed under the terms of the Creative Commons Attribution Noncommercial License (by-nc 4.0) which permits any noncommercial use, distribution, and reproduction in any medium, provided the original author(s) and source are credited. after experiencing during a basketball match an unprecedented sudden and unexpected syncope with retrograde amnesia and without loss of sphinterial function. Co-players reported a recovery time of approximately $2 \mathrm{~min}$. His own and family past medical history were largely unremarkable except for an aunt who died suddenly at the age of 20 (unfortunately it was impossible to retrieve further data regarding this sudden death since parents or relatives did not have clear memory of the dynamics of the event and in any case no autopsy was requested at that time).

Once admitted, the patient underwent computer tomography scan of the brain, electroencephalogram and a complete neurologic examination to rule out ischemic events or epilepsy. These tests came back with negative results. Medical physical examination showed normal systolic and diastolic blood pressure (110/70 $\mathrm{mmHg}$ ), heart rate of $70 \mathrm{bpm}$, body temperature of $36^{\circ} \mathrm{C}$, oxygen saturation on air at $99 \%$ and no signs or symptoms of heart failure.

A 12 leads electrocardiogram (ECG) showed regular sinus rhythm at $70 \mathrm{bpm}$ with T-wave inversion in the anterior leads (from V1 to V4), mild T-wave abnormalities in peripheral leads and incomplete right bundle branch block (Figure 1). No previous ECG of the patients were available.

Blood test revealed a mild increase in CPK, CPKMB, Myoglobin and troponin (220 U/L, $57 \mathrm{U} / \mathrm{L}, 813 \mathrm{ng} / \mathrm{mL}$ and 46.1 $\mathrm{ng} / \mathrm{L}$ respectively) likely as a result of intense physical activity. Hemoglobin, white blood cells, inflammation indexes, renal and liver function, and d-dimer were unremarkable.

A complete echocardiographic evaluation was performed and showed normal left ventricular (LV) size (end diastolic diameter: $45 \mathrm{~mm}$, end diastolic volume: $98 \mathrm{ml}$ ) and ejection fraction (EF: $56 \%$ ); normal thickness of the interventricular septum (10 mm) and posterior wall $(9 \mathrm{~mm})$ but with thinning of the apical segment of the lateral wall $(7 \mathrm{~mm})$ that appeared hypokinetic; right ventricle (RV) globally dilated with average transverse diameter of 44 $\mathrm{mm}$; dilatation of the outflow tract $(32 \mathrm{~mm})$; apical aneurysm, parietal bulging and spontaneous endocavitary ecocontrast; systolic function globally reduced with fractional area shortening of $38 \%$ and global strain - $10 \%$; evident trabeculation of the free wall with a "stack of plates" shape in mid-basal segment (Figure 2).

Given the above-mentioned data, the patient was admitted in our cardiology ward with the diagnosis of suspected cardiomyopathy.

The following day the patient underwent cardiac magnetic resonance (CMR) scan documenting the presence of severe RV dilatation and dysfunction ( $R V$ end diastolic volume $147 \mathrm{~mL} / \mathrm{m} 2$; $\mathrm{RVEF}=30 \%$ ). RV free wall thinning with diffuse hypokinesia and multiple dyskinetic areas (bulging) at RV outflow tract, inflow tract and apex were observed. Late enhancement in the diaphragmatic RV wall was present. Furthermore, LV involvement consist- 
ed in localized apical and lateral wall thinning and akinesia, fibrofatty infiltration of interventricular septum and lateral wall (Figures 3 and 4).

Given this clinical scenario, the patient underwent subcutaneous defibrillator (ICD) implantation. An episode of ventricular fibrillation was easily induced to test the device status and correctly recognized and terminated by the device. Of note, after the successful electric shock epsilon wave appeared on the ECG. It is likely that malignant arrhythmias might be the pathophysiological substrate leading to the clinical manifestation of the disease (syncope) (Figure 5).

These findings led us to the diagnosis of arrhythmogenic right ventricular cardiomyopathy (ARVC) with LV involvement. Sotalol $80 \mathrm{mg}$ daily was started accordingly.

\section{Discussion}

ARVC is a rare (in most cases inherited as an autosomal dominant genetic trait) cardiomyopathy firstly described in the 1970 s characterized by a progressive replacement of the myocytes by various degrees of fibro-adipose tissue involving particularly the areas between the anterior part of the pulmonary infundibulum, the apex, and the infero-posterior wall of the RV. Although up to $76 \%$ of the ARVC hearts studied at post-mortem disclosed a LV involvement (usually limited to the subepicardium or midmural layers of the postero-lateral free wall), the biventricular diseases is less common in vivo. These abnormalities can lead to sudden cardiac death and/or heart failure $[1,2]$.

Similarly to our case, T-wave inversions in the right precordial leads are present in up to $87 \%$ of adult patients with ARVC due to RV dilatation. However, they can be found in other contexts, such as in some young athletes, in ischemic cardiomyopathy, or during acute pulmonary embolism. Incomplete or complete right bundle branch block is also described and manifested in our patient. Epsilon wave (electrical potentials of small amplitude that occur at the end of the QRS complex and at the beginning of the ST segment in the right precordial leads) represents areas of delayed activation frequently localized in the RV as a consequence of fibrous and/or fibro-fatty replacement of myocardium and it is considered a major criterion for the diagnosis of ARVC $[1,3,4]$. Interestingly, in our patient epsilon wave was revealed only after defibrillation, likely as a result of a shock-induced massive increase of diastolic intracellular calcium concentration followed by activation of a transient sodium inward ion current [4,5].

The presence of segmental wall motion abnormalities (regional akinesia, dyskinesia, or dyssynchrony), combined with RV dilatation and/or dysfunction, are required for diagnosis, and were both present in our case. RV outflow tract dilatation and the reduction of RV fractional area are also part of the known criteria for the diagnosis of ARVC, but unfortunately they are usually seen in advanced stages. RV hypertrophic trabeculation is part of the structural abnormalities of the disease, and was clearly documented in our case. In addition, longitudinal strain derived from speckle tracking could be a sensitive tool for assessing regional and global myocardial function, particularly in early stages of the disease $[3,6,7]$.

Stress test must be considered to better understand the etiology of a syncope in young individuals occurred during sport activities [8]. Our case showed clearly an advanced RV disease that is likely to be the pathophysiologic substrate of the clinical manifestation

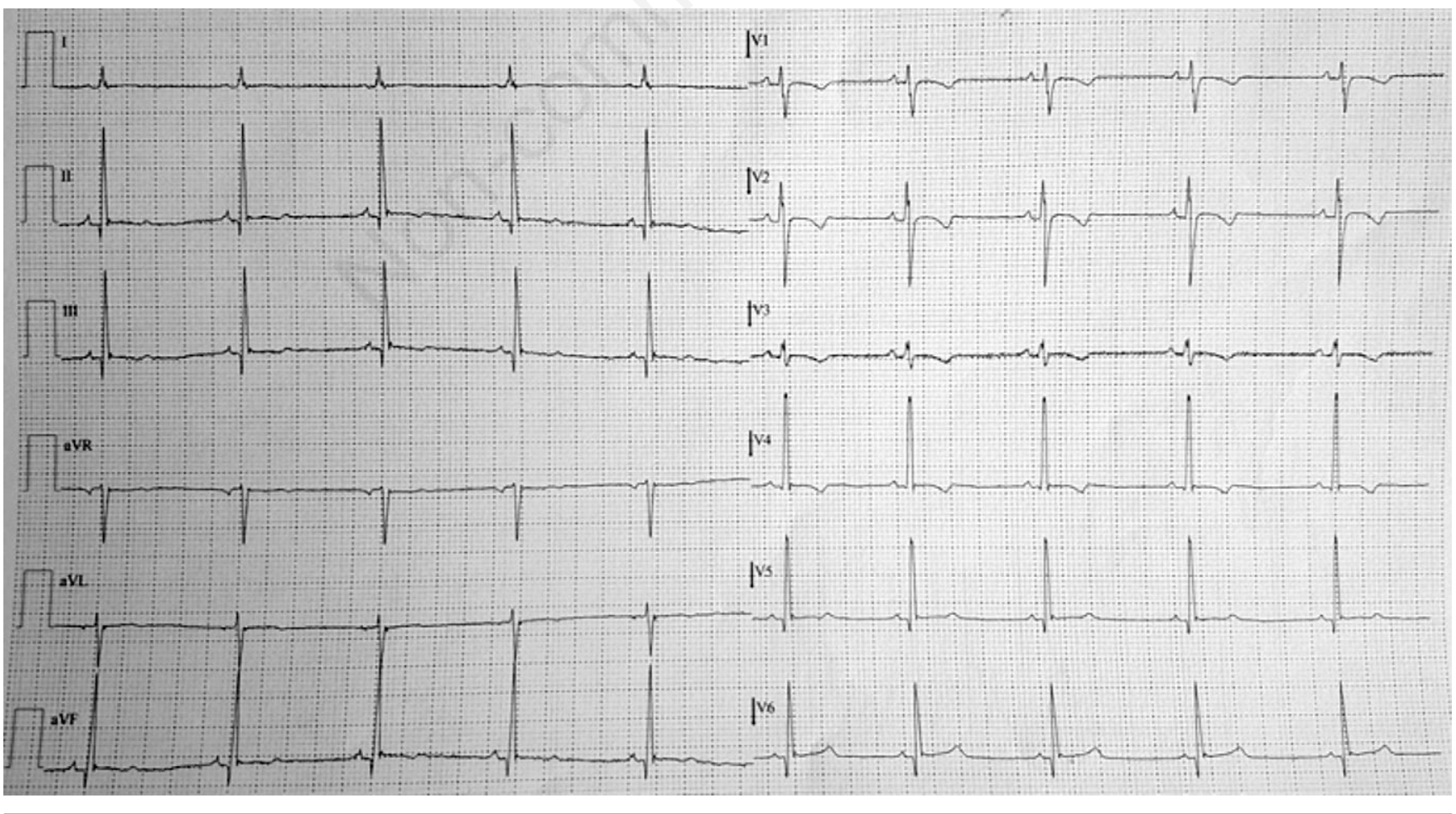

Figure 1. Baseline 12 leads ECG showing T-wave inversion from V1 to V4, mild T-wave abnormalities in peripheral leads and incomplete right bundle branch block. 
so we proceeded directly to the next level diagnostic test. In fact, CMR helped to confirm the diagnostic hypothesis and to characterize the extent of the disease, showing biventricular involvement that was less evident at the echocardiographic evaluation.

The diagnosis of ARVD remains challenging due to the absence of specific unique diagnostic criteria, its variable expressivity, and incomplete penetrance. In addition, although endomyocardial biopsies may help in the differential diagnosis (myocarditis, sarcoidosis and rarely Uhl's disease) they often generate nonspecific findings due to the patchy nature of the disease. Furthermore, safety concerns limit their use $[1,9,10]$. We all agreed that in our case this procedure was unnecessary and potentially harmful.

Genetic testing has reached dramatic improvement for the diagnosis of ARVC. To date, 16 genes have been associated with the ARVC phenotype, mostly those encoding desmosomal proteins [11]. We did not perform genetic screening since ECG, echo and CMR findings in our opinion were highly suggestive if not pathognomonic for the disease. However, genetic testing appears to be crucial to rule out ARVC in subjects in whom ECG, echocardiogram or CMR did not provide clear results and for screening pur-

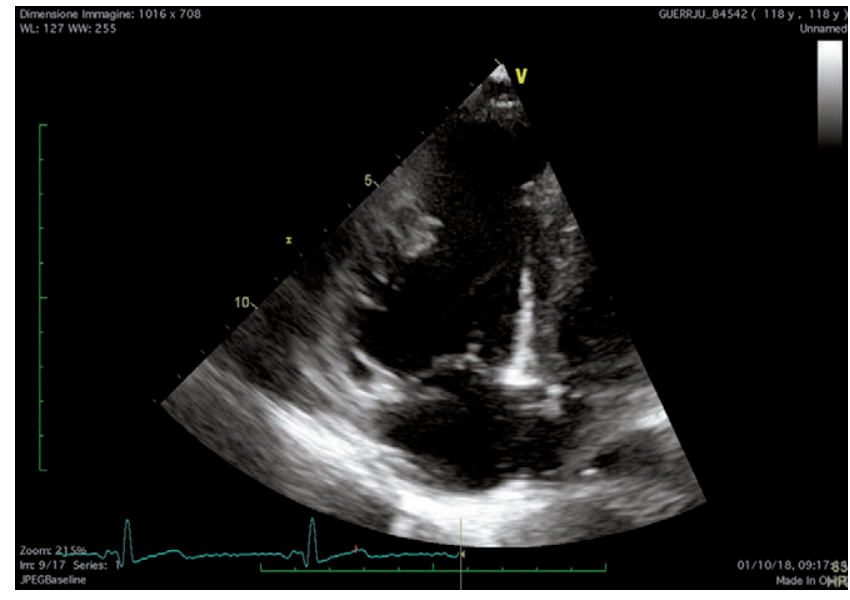

Figure 2. Apical 4C view showing RV parietal trabeculation and dilatation with "a pile stack" appearance in addition to apical aneurysm.

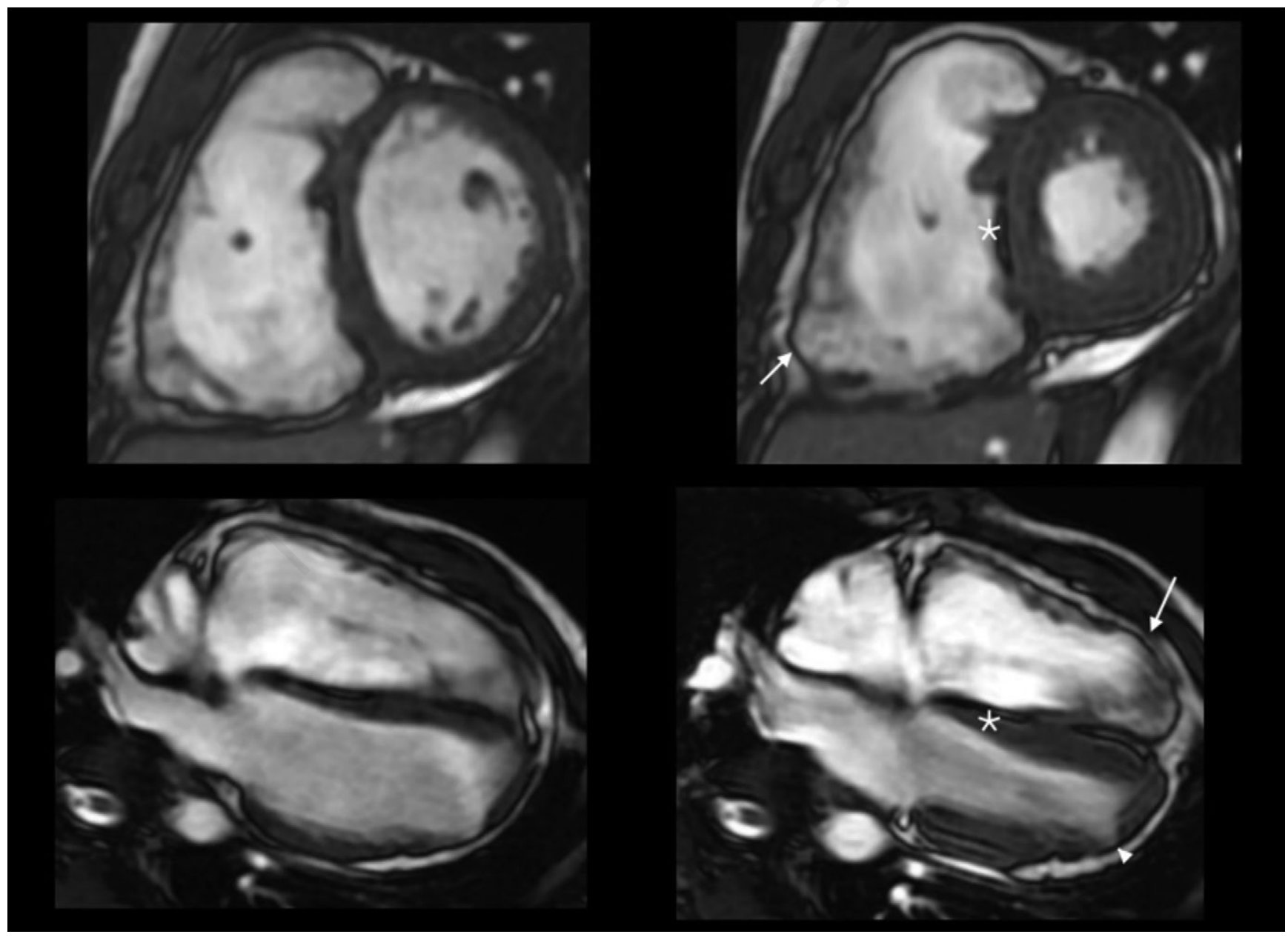

Figure 3. Short-axis (top panels) and four-chamber (bottom panels) FIESTA MRI images showing biventricular involvement. End-diastolic images are shown in the left panels, end-systolic images in the right panels. Free wall right ventricle thinning, and right ventricular dilatation are visible in both short axis and four-chamber views. Note sub-tricuspid and right ventricle apical bulging in end-systolic images (arrow). Localized left ventricle apical lateral wall thinning is also shown in end systolic four-chamber view (arrowhead). In all images localized adipose infiltration is evident in the right ventricular portion of mid inter ventricular septum (star). 
poses in parents or relatives of patients with diagnosed ARVC. Accordingly, we performed ECG and echocardiogram to the father and mother of our patient (57 and 48-year-old, respectively) and were within the normal limits (no further testing since at that age a manifestation of the disease is unlikely). Regarding the 20 -yearold asymptomatic sister, ECG and echocardiogram were equally normal, however, given the young age we decided to perform genetic screening fortunately with negative results.

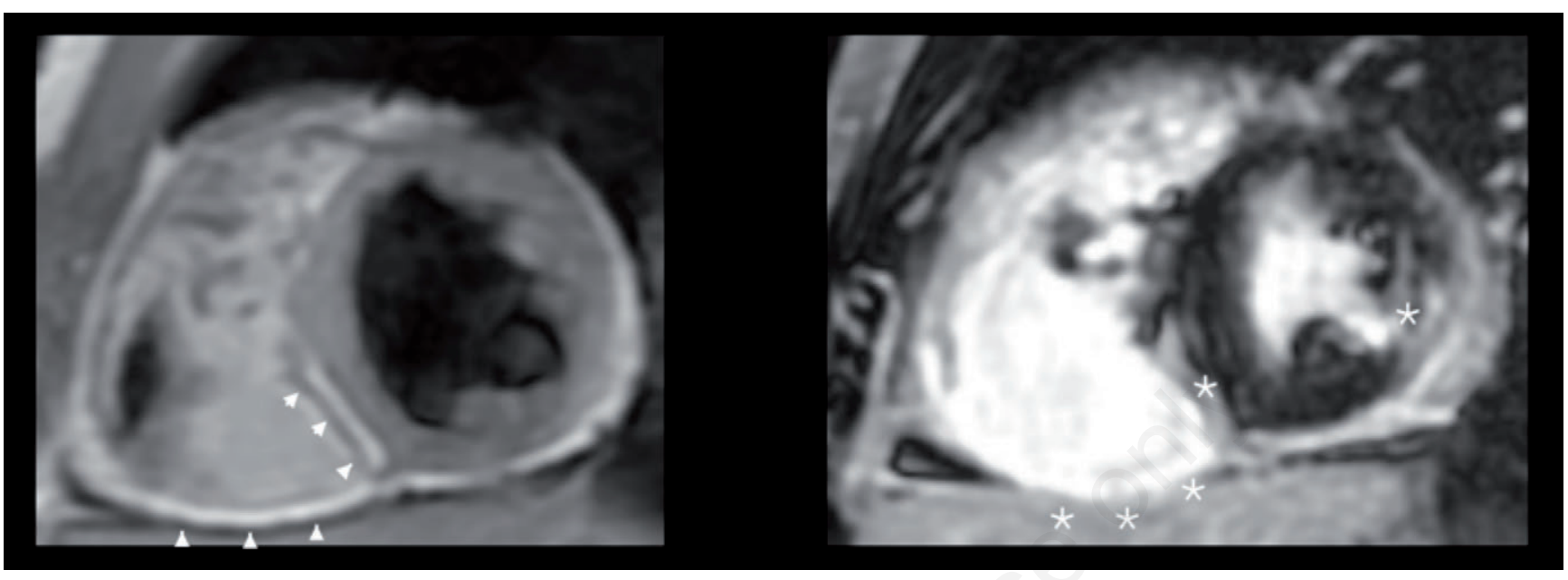

Figure 4. Short axis MRI images at apical level showing biventricular fibro-fatty infiltration in T1 black blood (left panel) and LGE images (right panel). In T1 images adipose infiltration of the right ventricular portion of mid interventricular septum is shown (arrow). LGE images: late enhancement of right ventricle diaframmatic wall (arrowhead), right portion of interventrular septum and subepicardial, intramyocardial late enhancement of left ventricle lateral wall (star) is showed.

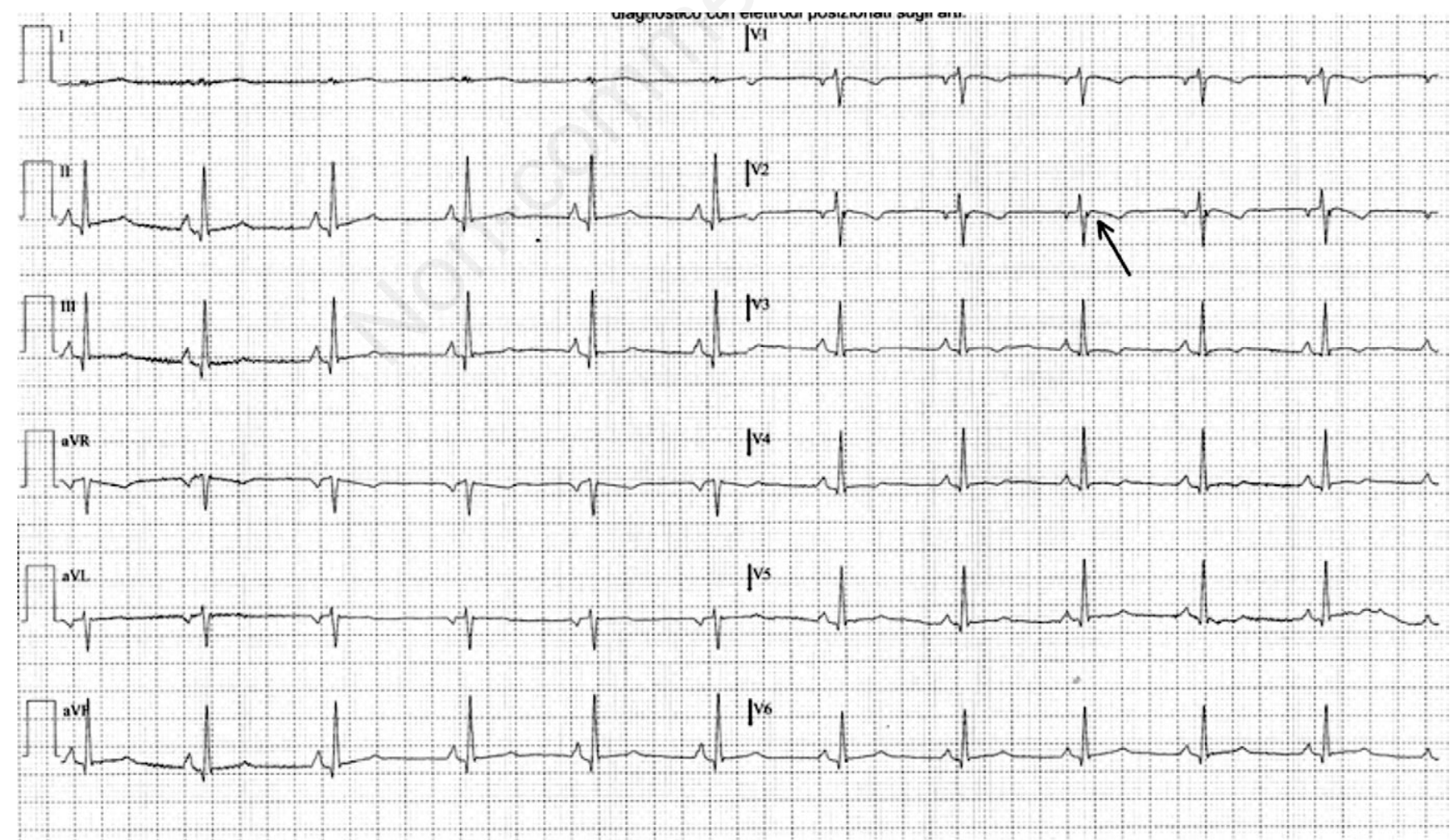

ECG: $10 \mathrm{~mm} / \mathrm{mV} .25 \mathrm{~mm} / \mathrm{s}$. Sitoln 0 . Letto n. $1.0 .05-40$

Figure 5. Post shock ECG showing epsilon wave in V2 not present at the baseline ECG (arrow). 


\section{Conclusions}

Our case depicts a typical onset of ARVC with syncope in a young, apparently healthy man during sport fatigue. Of note, despite the advanced disease according to echo and CMR results (biventricular involvement), this was the first clinical presentation at the age of 18. In fact, although the clinical course of the disease is known to be largely unpredictable, with such extensive morphological abnormalities malignant arrhythmias are often early symptomatic.

In general, ARVC must be suspected in case of right ventricular arrhythmias in young adults, a family history of ARVC or sudden death, or ECG abnormality such as T-wave inversion in the anterior leads. These abnormalities should guide physicians to perform other exams, such as echocardiogram ad eventually CMR to rule out the diagnosis of ARVC.

There is an unmet need to increase sensitivity and specificity of our tests as well as physicians' awareness to identify this patient population in order to decrease sudden death in young, otherwise healthy, adults.

\section{References}

1. Gandjbakhch E, Redheuil A, Pousset F, et al. Clinical diagnosis, imaging, and genetics of arrhythmogenic right ventricular cardiomyopathy/dysplasia: JACC State-of-the-art review. J Am Coll Cardiol 2018;72:784-804.

2. Corrado D, Basso C, Thiene G, et al. Spectrum of clinicopathologic manifestations of arrhythmogenic right ventricular cardiomyopathy/dysplasia: a multicenter study. J Am Coll Cardiol 1997;30:1512-20.
3. Marcus FI, McKenna WJ, Sherrill D, et al. Diagnosis of arrhythmogenic right ventricular cardiomyopathy/dysplasia: proposed modification of the task force criteria. Eur Heart $\mathrm{J}$ 2010;31:806-14.

4. Batchvarov VN, Bastiaenen R, Postema PG, et al. Novel electrocardiographic criteria for the diagnosis of arrhythmogenic right ventricular cardiomyopathy. Europace 2016;18:1420-6.

5. Jaoude SA1, Leclercq JF, Coumel P. Progressive ECG changes in arrhythmogenic right ventricular disease. Evidence for an evolving disease. Eur Heart J. 1996;17:1717-22.

6. Prakasa KR, Wang J, Tandri H, et al. Utility of tissue Doppler and strain echocardiography in arrhythmogenic right ventricular dysplasia/cardiomyopathy. Am J Cardiol 2007;100:507-12.

7. Te Riele AS, Tandri H, Sanborn DM, Bluemke DA. Noninvasive multimodality imaging in ARVD/C. J Am Coll Cardiol Img 2015;8:597-611.

8. Brignole M, Moya A, de Lange FJ, et al. ESC Scientific Document Group. 2018 ESC Guidelines for the diagnosis and management of syncope. Eur Heart J 2018;39:1883-948.

9. Pieroni M, Dello Russo A, Marzo F, et al. High prevalence of myocarditis mimicking arrhythmogenic right ventricular cardiomyopathy differential diagnosis by electroanatomic mapping-guided endomyocardial biopsy. J Am Coll Cardiol 2009; 53:681-9.

10. Ott P, Marcus FI, Sobonya RE, et al. Cardiac sarcoidosis masquerading as right ventricular dysplasia. Pacing Clin Electrophysiol 2003;26:1498-503.

11. Awad MM, Calkins H, Judge DP. Mechanisms of disease: molecular genetics of arrhythmogenic right ventricular dysplasia/cardiomyopathy. NatClin Pract Cardiovasc Med 2008;5: 258-67. 